Faculdade de Ciências Econômicas UFRGS
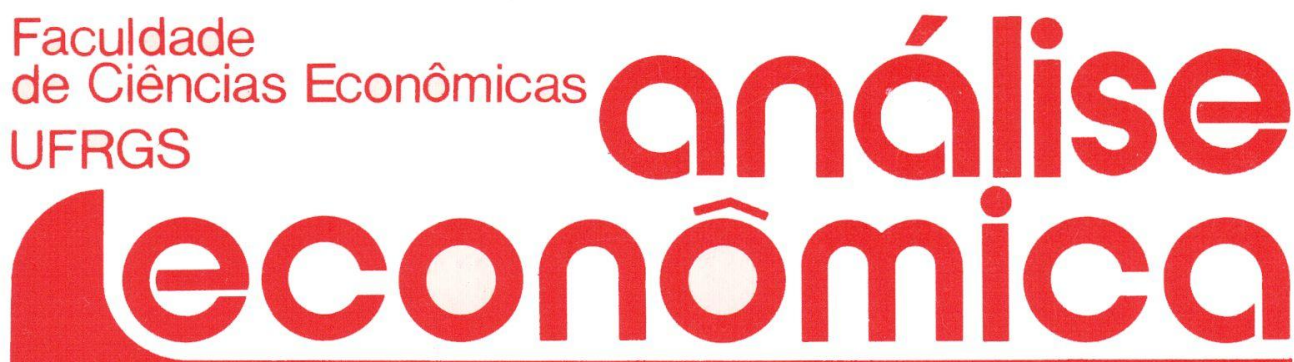

- A DIVIDA EXTERNA: UMA CHANCE DE SOLUÇÃO?

James Kenneth Galbraith

- A ECONOMIA BRASILEIRA VISTA DE FORA

Hartmut Sangmeister

- A QUESTÃO DO INVESTIMENTO Hélio Nogueira da Cruz

- EXCESSO DE CAPACIDADE NA CONCORRÊNCIA MONOPOLISTICA João Rogério Sanson

- MODELOS LINEARES QUADRÁTICOS

Gláucia de Oliva Pretto

- A PEQUENA PROdUÇÃo AGRÍCOLA

Silvana Saionara Gollo

Otto Guilherme Konzen

- ÍNDICE DE PREÇOS AO CONSUMIDOR: UM ESTUDO SOBRE SUA DETERMINAÇÃO NO BRASIL

Neio Lúcio Peres Gualda
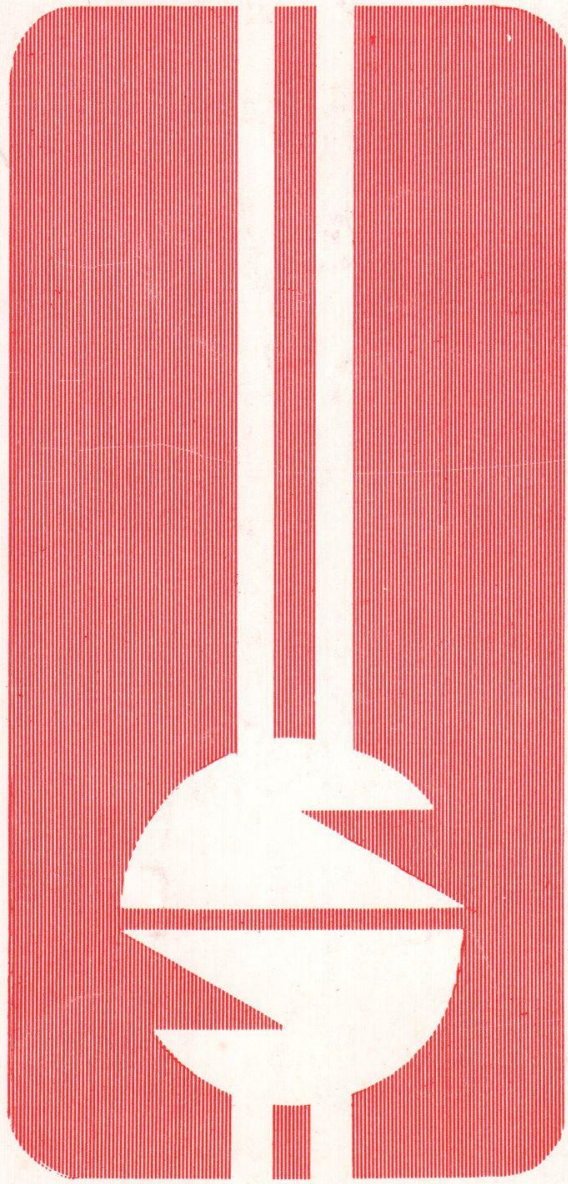
UNIVERSIDADE FEDERAL DO RIO GRANDE DO SUL

Reitor. Prof. Gerhard Jacob

FACULDADE DE CIÊNCIAS ECONÔMICAS

Diretor. Prof. Walter Meucci Nique

CENTRO DE ESTUDOS E PESQUISAS ECONÔMICAS

Diretor: Prof. Reinaldo Ignácio Adams

DEPARTAMENTO DE CIÉNCIAS ECONÔMICAS

Chefe: Prof- Ottlia Beatriz Kroeff Carrion

CURSO DE PÓS-GRADUAÇĀO EM ECONOMIA

Coordenador. Prof. Roberto Camps Moraes

CURSO DE PÓS-GRADUAÇĀO EM ECONOMIA RURAL

Coordenador. Prof. Aray Miguel Feldens

CONSELHO EDITORIAL:

Achyles Barcelos da Costa, Aray Miguel Feldens, Atos Freitas Grawunder, Carlos Augusto Crusius, Ernani Hickmann, Humberto Vendelino Richeter, João Rogério Sanson, Juvir Luiz Mattuella, Maria Imilda da Costa e Silva, Nali de Jesus de Souza, Nuno Renan Lopeś de Figueiredo Pinto, Otllia Beatriz Kroeff Carrion, Otto Guilherme Konzen, Paulo Alexandre Spohr, Pedro Cezar Dutra Fonseca, Reinaldo Ignácio Adams, Roberto Camps Moraes, Valter José Stülp, Yeda Rorato Crusius e Edgar Augusto Lanzer (UFSC).

COMISSÃO EDITORIAL: Atos Freitas Grawunder, Pedro Cezar Dutra Fonseca, Reinaldo Ignácio Adams e Roberto Camps Moraes.

EDITOR: Nali de Jesus de Souza

SECRETARIA: Zélide Bregalda, Francisco Carlos Becco dos Santos, Vanete Ricacheski (revisão de textos), Maria Ivone de Mello (normalização)

FUNDADOR: Prof. Antônio Carlos Rosa

A Revista ANÁLISE ECONÔMICA publica dois números anuais nos meses de março e novembro. O preço da assinatura, para 1989, é de 7,5 BTNs (o núrnero avulso custa 4 BTNs), a ser pago através de cheque nominal em nome da "Faculdade de Ciências Econômicas/ UFRGS". Aceita-se permuta com revistas congêneres. Aceitam-se, também, livros para elaboração de resenhas ou recensões.

Toda correspondência, material para publicação, assinaturas e permutas devern ser dirigidos ao seguinte destinatário:

$$
\begin{gathered}
\text { PROF. NALI DE JESUS DE SOUZA } \\
\text { Revista Análise Econômica } \\
\text { Av. João Pessoa, } 52 \text { - sala } 28 \\
90.040 \text { - PORTO ALEGRE (RS) - BRASIL } \\
\text { Telefone: } 25-5844 \text { ramal } 34
\end{gathered}
$$




\title{
PLANEJAMENTO ÓTIMO: UMA ABORDAGEM SOBRE MODELOS LINEARES QUADRÁTICOS
}

\author{
Gláucia de Oliva Pretto *
}

\section{SINOPSE}

Este artigo tem por objetivo verificar como a teoria de controle Stimo pode ser inserida em um processo de planejamento econômico, a fim de se obter uma seqüência de polfticas "ótimas", isto é, um vetor de polfticas cujos niveis serão frutos de um processo de otimização dinâmica. Foi analisada a metodologia de um modelo linear quadrático obtendo-se relevantes interpretaçð̄es económicas, atravês das variáveis co-estado:

Ficou evidente a necessidade de se pensar na especificação de modelos econométricos com vistas à sua aplicação na teoria de controle stimo, para que se possa usufruir de todo o potencial de análise, dado pela regra de controle e pelos multiplicadores de Lagrange.

Nos dias de hoje, é comumente aceito pela sociedade que, cabe ao Estado, o exercício de um razoável controle da vida econômica de um pals. Tal tarefa, sem dúvida, constitui-se em um processo tanto abrangente quanto complexo, devido às inúmeras decisōes entre politicas alternativas, que o Governo pode tomar.

$\mathrm{Na}$ investigação de um caminho que exerça influência nas relações entre os agentes econômicos, e que seja coerente com os objetivos pretendidos, vários instrumentos de Programação Econômica foram desenvolvidos. Entre eles, destacam-se os modelos econométricos dinâmicos de equaçóes simultâneas associados à simulação computacional que possibilitam a comparação de diferentes políticas, facilitando a escolha daquela que, entre as testadas, mais aproxima o sis-

- Professora do Departamento de Estatfstica e do Curso de Pós-Graduaçăo em Economia Rural da UFRGS; pesquisadora do IEPE/UFRGS.

ANÁLISE ECONÔMICA

ANO 6

№ 10

NOVEMBRO/88

p.63-86 
tema econômico dos propósitos pretendidos. Esse caminho, no entanto, é insuficiente na pesquisa de um "plano śtimo" de intervenção estatal. Por tentativa e erro, pode-se, no máximo, encontrar uma seqüência de políticas que, sem ser necessariamente ótima, aproxima a economia das aspirações da sociedade.

Para se obter um vetor de politicas ótimas, é necessário que o planejamento econômico seja considerado como úm problema de otimização dinâmica. Para tanto, os planejadores deverão reunir metas e restriçōes do sistema na forma de uma função-objetivo. Tal função deverá traduzir, direta ou indiretamente, o bem-estar social para ser otimizado em um perlodo de tempo determinado. Esse tipo de modelo tem, como fundamento, a escolma de trajetórias temporais ótimas para as variáveis que estāo sujeitas ao controle do Governo (variáveis de controle ou de polftica), visando, através delas, a alcançar determinadas metas para as variáveis consideradas relevantes à vida econômica e social de um pals.

Muito embora o interesse pelo planejamento macroeconômico seja ainda recente, algumas técnicas de otimização dinâmica começam a despontar na literatura econômica. Entre elas, citam-se os elementos do Cálculo Variacional, o Princlpio do Máximo de Pontryagin e a Programação Dinâmica. Este ramo de conhecimento, que se constitui em métodos matemáticos avançados, recebe, na literatura pertinente, a denominação de Teoria de Controle Ótimo. Apesar da importância da Teoria de Controle Ótimo na Teoria Econômica, os métodos de resolução não são facilmente encontrados em bibliografia nacional. Além disso, constituem-se em complexos desenvolvimentos matemáticos que, em sua quase totalidade, são apresentados considerando o tempo como uma variável contínua.

Em versão discreta, dois métodos de resolução de modelos de controle ótimo estão disponlveis na bibliografia especializada: a Programação Dinâmica e o Modelo Linear Quadrático (MLQ). O primeiro não possui um algoritmo próprio de solução, ele é resolvido pelo Princlpio Geral de Bellman; o segundo, no entanto, pode ser resolvido por meio de um algoritmo programávet através do qual obtém-se relevantes interpretações econômicas.

Este artigo tem por objetivo mostrar como um Modelo Linear Quadrático pode ser utilizado em um plano de intervenção estatal para que se possa avaliar os efeitos quantitativos de politicas altemativas de estabilização econômica. 


\section{1 - O MODELO LINEAR QUADRÁTICO MAIS SIMPLES}

O procedimento metodológico, que será apresentado, visa a descrever o contexto dentro do qual a Teoria de Controle Ótimo pode ser usada na seleçāo de uma política governamental, capaz de levar o sistema econômico aos fins pretendidos pela sociedade. Isto é, uma seqüência de polfticas que nāo será mais arbitrariamente determinada pelos planejadores. Seus niveis serão frutos de um processo de otimização dinâmica.

\section{1 - DEFINIÇÃO E DESENVOLVIMENTO}

Um Modelo Linear Quadrático trata de uma função-objetivo quadrática e de um conjunto de restriçōes, formando um sistema de equaçōes a diferenças. A função-objetivo representa a soma dos quadrados dos desvios entre os valores observados das variáveis-estado e suas metas. Associando-se à função-objetivo um sistema linear de equaçōes a diferenças tem-se a configuração de um MLQ, que é dada por.

Otimizar $\quad D=\frac{1}{2} \sum_{t_{0}}^{t_{f}}\left(Y_{t}-m_{t}\right)^{\prime} P_{t}\left(Y_{t}-m_{t}\right)$

$x_{\text {to }} \ldots x_{\mathrm{tf}}$

Sujeito a $Y_{t}=A_{t} Y_{t-1}+C_{t} X_{t}+b_{t}$

onde:

$t_{0}=$ tempo inicial;

$t_{f}=$ tempo final;

$Y_{t}=$ vetor $\mathrm{px}_{1}$ das variáveis estado do sistema no perlodo $t$;

$P_{t}=$ matriz diagonal de pesos de ordem $p$ no perlodo $t ;$

$m_{t}=$ vetor $\mathrm{px}_{1}$ de metas para as variáveis estado no período $t$;

$\mathrm{x}_{\mathrm{t}}=$ vetor $\mathrm{qx}_{1}$ das variáveis de controle do sistema no perlodo $\mathrm{t}$; 
$A_{t}=$ matriz de ordem $p$ de coeficientes das variáveis-estado no período t;

$C_{1}=$ matriz de ordem pxq de coeficientes das variáveis de controle no perlodo t;

$b_{1}=$ vetor $\mathrm{px}_{1}$ das variáveis exógenas não sujeitas a controle e termos constantes no perlodo t.

A matriz $P_{t}$ é uma matriz diagonal, cujos elementos sāo ponderaçōes atribuldas a cada variável-estado. É utilizada para atribuir graus relativos de importância à atingibilidade das metas especificadas para cada uma dessas variáveis.

As metas consistem na especificação de valores que as variáveis endógenas do modelo deveriam, idealmente, atingir durante um perlodo de planejamento - de um tempo inicial $t_{o}$ a um tempo final $t_{f}$.

A resolução do MLQ é desenvolvida de modo análogo ao de otimizaçāo estática. A função de Lagrange do problema é:

$D_{1}=\Sigma_{t_{0}}^{t_{f}} \quad\left(Y_{t}-m_{t}\right)^{\prime} P_{t}\left(Y_{t}-m_{t}\right)-\lambda_{t}^{\prime}\left(Y_{t}-A_{t} Y_{t-1}-C_{t} X_{t}-b_{t}\right)$

Igualando a zero as derivadas de $D_{1}$ vem:

$$
\begin{aligned}
& P_{t}\left(Y_{t}-m_{t}\right)-\lambda_{t}+A^{\prime}{ }_{t+1} \lambda_{t+1}=0 \\
& C^{\prime}{ }_{t} \lambda_{t}=0 \\
& -\left(Y_{t}-A_{t} Y_{t-1}-C_{t} X_{t}-b_{t}\right)=0
\end{aligned}
$$

As equações (2) a (4) constituem um sistema de incóginitas $Y_{t}, X_{t}$ e t, para $t=1,2, \ldots, t_{f}$. A solução deste sistema pode ser obtida recursivamente em três etapas, iniciando-se em $t_{f}$ até chegar-se a $t_{o}{ }^{1}$.

$\mathrm{Na}$ primeira etapa, expressa-se o multiplicador dinâmico de Lagrange, $\lambda_{t}$, como uma função de $Y_{t}$, por meio da equação.

\footnotetext{
1 Esse desenvolvimento é baseado em CHOW (1975, p. 156-60.)
} 


$$
P_{t f} Y_{t f}-P_{t f} m_{t f}-\lambda_{t f}+A^{\prime}{ }_{t+1} \lambda_{t f+1}=0 \operatorname{com} \lambda_{t f+1}=0
$$

logo:

$$
\begin{aligned}
& \lambda_{\mathrm{tf}}=P_{\mathrm{tf}} Y_{\mathrm{tf}}-P_{\mathrm{tf}} \mathrm{m}_{\mathrm{tf}} \quad \text { ou } \\
& \lambda_{\mathrm{tf}}=\mathrm{H}_{\mathrm{tf}} Y_{\mathrm{tf}}-\mathrm{P}_{\mathrm{tf}} \mathrm{m}_{\mathrm{tf}},
\end{aligned}
$$

onde $H_{t f}$ e $h_{t f}$ são as condições iniciais dadas por:

$$
\begin{aligned}
& H_{t f}=P_{t f} \\
& h_{t f}=P_{t f} m_{t f}
\end{aligned}
$$

$\mathrm{Na}$ segunda etapa, procura-se uma equação que relacione os niveis das variáveis de controle com os das variáveis-estado. $\dot{E}$ a denominada "equação ou regra de controle" que é obtida em duas passagens: primeiro, substitui-se (5) em (3), obtendo-se:

$$
C_{t f}^{\prime}-\left(H_{t f} H_{t f}-h_{t f}\right)=0 \text {. }
$$

Substituindo-se, agora, (4) em (8) vem:

$$
C_{t f}^{\prime}\left[H_{t f}\left(A_{t f} Y_{t f-1}+C_{t f} X_{t f}+b_{t f}\right)-h_{t f}\right]=0
$$

ou, após rearranjo de formas:

$C^{\prime}{ }_{t f} H_{t f} A_{t f} Y_{t f-1}+C^{\prime}{ }_{t f} H_{t f} C_{t f} X_{t f}+C^{\prime}{ }_{t f} H_{t f} b_{t f}-C^{\prime}{ }_{t f} h_{t f}=0$ donde:

$$
\begin{aligned}
X_{t f}= & -\left(C^{\prime}{ }_{t f} H_{t f} C_{t f}\right)^{-1}\left(C^{\prime}{ }_{t f} H_{t f} A_{t f}\right) Y_{t f-1}-\left(C^{\prime}{ }_{t f} H_{t f} C_{t f}\right)-1 \\
& C^{\prime}{ }_{t f}\left(H_{t f} b_{t f}-h_{t f}\right)
\end{aligned}
$$

que é a regra de controle procurada, cuja forma sintética é:

$$
X_{t f}=L_{t f} Y_{t f-1}+r_{t f}
$$


onde:

$$
\begin{aligned}
& L_{t f}=-\left(C^{\prime} H_{t f} C_{t f}\right)^{-1}\left(C^{\prime} H_{t f} H_{t f} A_{t f}\right) \\
& r_{t f}=-\left(C^{\prime} H_{t f} H_{t f} C_{t f}\right)^{-1} C_{t f}^{\prime}\left(H_{t f} b_{t f}-h_{t f}\right) .
\end{aligned}
$$

A última etapa desse desenvolvimento resolve $Y_{t f}$ em funçāo de $Y_{t f-1}$. Para tanto, substitui-se (9) em (4) obtendo-se:

$$
Y_{t f}=\left(A_{t f}+C_{t f} L_{t f}\right) Y_{t f-1} C_{t f} f_{t f}+b_{t f}
$$

Ainda, expressa-se o valor de $\lambda_{t f}$, também, em função de $Y_{t f-1}$, por meio da substituição de (12) em (5), isto é:

$$
\begin{aligned}
& \lambda_{t f}=H_{t f} Y_{t f}-h_{t f} \\
& \lambda_{t f}=H_{t f}\left[\left(A_{t f}+C_{t f} L_{t f}\right) Y_{t f-1}+C_{t f} m_{t f}+b_{t f}\right]-h_{t f} \\
& \lambda_{t f}=H_{t f}\left(A_{t f} C_{t f} L_{t f}\right) Y_{t f-1}+H_{t f} C_{t f} m_{t f}+H_{t f} b_{t f}-h_{t f}
\end{aligned}
$$

Com (13) em (2) consegue-se uma equação análoga à (5) da primeira etapa, ou seja:

$$
\lambda_{\mathrm{tf}-1}=\mathrm{P}_{\mathrm{tf}-1} Y_{\mathrm{tf}-1}-\mathrm{P}_{\mathrm{tf}-1} \mathrm{~m}_{\mathrm{tt}-1}+\mathrm{A}_{\mathrm{tf}}^{\prime} \lambda_{\mathrm{tf}}
$$

ou

$$
\lambda_{t f-1}=H_{t f-1} Y_{t f-1}-h_{t f-1}
$$

donde:

$$
\begin{aligned}
& H_{t f-1}=P_{t f-1}+A^{\prime}{ }_{t f} H_{t f}\left(A_{t f}+C_{t f f} L_{t f}\right) \\
& h_{t f-1}=P_{t f-1} m_{t f-1}-A^{\prime}{ }_{t f} H_{t f}\left(b_{t f}+C_{t f} r_{t f}\right)+A^{\prime}{ }_{t f} h_{t f}
\end{aligned}
$$

Essas três etapas de solução sāo aplicadas ao problema utilizando-se o par de equações (10) e (14) para o cálculo de $L_{t f}, H_{t f-1}$, $L_{\mathbb{H}}-1, \ldots, H_{1}, L_{1}$. Os cálculos devem levar em consideração a condição inicial dada por $\mathrm{H}_{\mathrm{tf}}=\mathrm{P}_{\mathrm{tf}}$. Tem-se, nesse caso, a seguinte seqüência de cálculos: 


$$
\begin{aligned}
& L_{t f}=-\left(C^{\prime}{ }_{t f} H_{t f} C_{t f}\right)^{-1}\left(C^{\prime}{ }_{t f} H_{t f} A_{t f}\right) \\
& H_{t f-1}=P_{t f-1}+A^{\prime}{ }_{t f} H_{t f}\left(A_{t f}+C_{t f} L_{t f}\right) \\
& L_{t f-1}=\left(C^{\prime}{ }_{t f-1} H_{t f-1} C_{t f-1}\right)^{-1}\left(C^{\prime}{ }_{t f-1} H_{t f-1} A_{t f-1}\right) \\
& \ldots \ldots \ldots \ldots \ldots \ldots \ldots \ldots \ldots \ldots \ldots \ldots \ldots \ldots \ldots \ldots \ldots \ldots \ldots \ldots \ldots \ldots \ldots \ldots \\
& H_{1}=P_{1}+A^{\prime}{ }_{2} H_{2}\left(A_{2}+C_{2} L_{2}\right) \\
& L_{1}=\left(C^{\prime}{ }_{1} H_{1} C_{1}\right)^{-1}\left(C^{\prime}{ }_{1} H_{1} A_{1}\right) .
\end{aligned}
$$

Obtidas as matrizes $H_{t}$ usa-se o par de equaçōes (11) e (15) para calcular os vetores $r_{f f}, h_{t f}, r_{t f-1} \ldots, r_{1}$ e $h_{1}$, considerando, também neste caso, a condição inicial $h_{t f}=P_{t f} m_{t f}$. A seqüência de cálculos é a seguinte:

$$
\begin{aligned}
& r_{t f}=-\left(C^{\prime}{ }_{t f} H_{t f} C_{t f}\right)^{-1} C^{\prime}{ }_{t f}\left(H_{t f} b_{t f}-h_{t f}\right) \\
& h_{t f-1}=P_{t f-1} m_{t f-1}-A^{\prime}{ }_{t f} H_{t f}\left(b_{t f}+C_{t f t} r_{t f}\right)+A^{\prime} h_{t f} \\
& r_{t f-1}=-\left(C^{\prime}{ }_{t f-1} H_{t f-1} C_{t f-1}\right)^{-1} C^{\prime}{ }_{t f-1}\left(H_{t f-1} b_{t f-1}-h_{t f-1}\right)
\end{aligned}
$$

$$
\begin{aligned}
& h_{1}=p_{1} m_{1}-A^{\prime}{ }_{2} H_{2}\left(b_{2}+C_{2} r_{2}\right)+A^{\prime}{ }_{2} h_{2} \\
& r_{1}=-\left(C^{\prime}{ }_{1} H_{1} C_{1}\right)^{-1} C^{\prime}{ }_{1}\left(H_{1} b_{1}-h_{1}\right) .
\end{aligned}
$$

A partir do conhecimento das matrizes $\iota_{t}$ e $H_{t}$ e dos vetores $r_{t} e$ $h_{t}$ fica determinada a trajetória ótima das variáveis de controle, $X_{t}$, dada pela equação de controle, (9). Ficam, determinados, também, os multiplicadores de Lagrange dados por (5).

A solução do problema pode ser sintetizada através das seguintes fases de cálculos para $t=1,2, \ldots, t_{f}$.

1 Fase: $L_{t}=-\left(\mathrm{C}^{\prime}{ }_{t} \mathrm{H}_{t} \mathrm{C}_{t}\right)^{-1} \mathrm{C}^{\prime}{ }_{t} \mathrm{H}_{t} \mathrm{~A}_{t}$

2a Fase: $H_{t}=P_{t}+A_{t+1}^{\prime} H_{t+1}\left(A_{t+1} C_{t+1} L_{t+1}\right)$

69 


$$
\begin{aligned}
& \text { 3' Fase: } r_{t}=-\left(C^{\prime}{ }_{t} H_{t} C_{t}\right)^{-1} C^{\prime}{ }_{t}\left(H_{t} b_{t}-h_{t}\right) \\
& \text { 4. Fase: } h_{t}=P_{t} m_{t}-A_{t+1}^{\prime} H_{t+1}\left(b_{t+1}+C_{t+1} r_{t+1}\right)+A_{t+1}^{\prime} h_{t+1} \\
& \text { 5 Fase: } Y_{t}=\left(A_{t}+C_{t} L_{t}\right) Y_{t-1}+C_{t} m_{t}+b_{t} \\
& \text { 6 Fase: } X_{t}=L_{t} Y_{t-1}+m_{t} \\
& 7^{\mathrm{a}} \text { Fase: } \lambda_{\mathrm{t}}=\mathrm{H}_{\mathrm{t}} \mathrm{Y}_{\mathrm{t}}-\mathrm{h}_{\mathrm{t}} \text {. }
\end{aligned}
$$

O principal aspecto desse algoritmo refere-se à sexta fase de cálculo. Nos modelos usuais, as variáveis exógenas sảo determinadas fora do sistema. Aqui, mesmo constituindo um subconjunto das exógenas, seus niveis são dados pela equação de controle. Esse tipo de resultado traduz-se em uma das vantagens desse método de solução, pois uma solução analítica desse tipo dificilmente é observada em modelos de otimização dinâmica.

Um simples exame da regra de controle, $X_{t}=L_{t} Y_{t-1}+r_{t}$, mostra que as variáveis de política são influenciadas pelos niveis passados das variáveis-estado (endógenas ao sistema). Esta equação esclarece a relação existente entre endógenas defasadas e controles correntes. Mais ainda, os graus relativos de importância atribuildos às metas de cada variável-estado influenciam, a cada perlodo, os niveis das variáveis de controle, através das matrizes de coeficientes $L_{t}$ e $r_{t}$.

É interessante observar que a solução de um MLQ pode ser obtida pelo método tradicional de soluçāo de sistemas de equacōes simultâneas. Para tal realização, as equaçōes (2) a (4) são tomadas simultaneamente como um sistema linear de incógnitas $X_{t}, Y_{t} e_{t}$ para $t$ $=1,2, \ldots, t_{f}$. Seu desenvolvimento, todavia, é muito trabalhoso pois existirão $t_{f}(q+2 p)$ dessas equaçōes. Além disso, com esse modo de solução, perde-se a "equação de controle" que constitui o fundamento do MLQ, como procedimento metodologico para avaliar o planejamento econômico no contexto da Teoria de Controle Ótimo.

Além dessas observaçōes, deve-se ainda destacar o significado e a utilidade dos multiplicadores de Lagrange (variáveis co-estado) em problemas de estabilização econômica.

Como se sabe, essas variáveis são interpretadas como "preçossombra". Aqui, no MLQ, o significado não é diferente; elas represen- 
tam o custo marginal resultante de um desvio entre a correspondente variávelestado e sua respectiva meta.

A importância das variáveis co-estado reside no fato de senvirem de indicadores das variáveis mais relevantes para o custo de uma polftica ótima. Elas mostram, por exemplo, que pequenas variaçōes nas condiçỏes iniciais de alguma variável-estado têm grande impacto no custo total mínimo. Ou, que este pode ser muito sensivel aos valores de determinadas variáveis em alguns pontos do tempo e, a outras, em outros pontos. Mais ainda, através delas pode-se avaliar as conseqüências de comportamentos subótimos do sistema, que podem advir de choques aleatórios, incapacidade política para a implementação dos controles ótimos ou de modelos econométricos inadequados. Nesse sentido, os multiplicadores de Lagrange indicam quando poderá existir algum grau de flexibilidade em um programa de estabilização econômica. Isto é, em que pontos do tempo será essencial forçar determinadas variáveis a permanecerem em suas trajetórias ótimas, enquanto que outras poderão distanciar-se delas sem muito comprometer os resultados ótimos.

Nesse contexto, diferentes trajetórias subótimas, correspondentes a diferentes matrizes de pesos, podem ser avaliadas. Isso porque, para cada matriz de pesos, $P_{t}$, tem-se uma trajetória ótima para as variáveis co-estado. Mas se houver impossibilidade de o sistema econômico trilhar o caminho ótimo, pode-se verificar quais metas devem ser prioritárias. Isso tudo com o objetivo de se obter uma trajetória ótima, cujo caminho subótimo implique menor custo. Pode-se fazer isso, simulando o sistema para diversas combinaçōes de pesos, a fim de escolher aquela que apresentar os menores valores para os multiplicadores de Lagrange.

Como se vê, além do algoritmo de solução, que propicia a equação de controle, obtém-se relevantes interpretações econômicas dadas pelas variáveis co-estado.

\section{2 - REQUISITOS METODOLÓGICOS}

O processo de solução do MLQ foi apresentado de forma generalizada. Nele foi considerado o caso no qual as matrizes de coeficientes $A_{t}$ e $C_{t}$ são variáveis ao longo do período de planejamento. 
$\dot{E}$ útil ressaltar ainda, o caso em que as matrizes $A$ e $C$ são constantes durante o periodo considerado, em conjunto com a circunstância da não-singularidade das matrizes $\mathrm{Ce} \mathrm{H}_{\mathrm{t}}$.

Seguindo as mesmas fases de cálculo da seção anterior observase que:

Fase l: A matriz de coeficientes $L_{t}$ dada por.

$$
L_{t}=-\left(C^{\prime} H_{t} C\right)^{-1} C^{\prime} H_{t} A \text { torna-se uma constante para todo o }
$$
perlodo de planejamento:

$$
\begin{aligned}
& L_{t}=C^{-1} H_{t}^{-1} C^{\prime}-1 C^{\prime} H_{t} A \\
& L_{t}=C^{-1} A \quad \text { para todo } t=1,2, \ldots, t_{f}
\end{aligned}
$$

Isso significa que, os pesos atribuídos às metas estipuladas para as variáveis estado, não exercerão influência nos demais periodos de planejamento. Conforme visto anteriormente, essa influência é levada adiante via matriz $H_{t}$, que, nesse caso, desaparece do cálculo de $L_{t}$. Assim, a matriz de coeficientes das endógenas defasadas da regra de controle, (9), será sempre a mesma.

Fase II: As matrizes, $H_{t}$, tornam-se iguais às matrizes de pesos $P_{t}$ :

$$
\begin{aligned}
& H_{t}=P_{t}+A^{\prime} H_{t+1}\left(A+C L_{t}\right) \\
& H_{t}=P_{t}+A^{\prime} H_{t+1}\left(A-C C^{-1} A\right) \\
& H_{t}=P_{t}+A^{\prime} H_{t+1}(A-A) \\
& H_{t}=P_{t} .
\end{aligned}
$$

Fase III: $\mathrm{O}$ vetor $r_{\mathrm{t}}$ fica independente dos pesos

$$
r_{t}=-\left(C^{\prime} H_{t} C\right)^{-1}\left(H_{t} b_{t}-h_{t}\right)
$$

mas, como $\mathrm{C}$ e $\dot{H}_{\mathrm{t}}$ são não-singulares por hipótese, vem:

$$
r_{t}=-C^{\prime} H_{t}-1 C^{\prime}-1\left(H_{t} b_{t}-h_{t}\right)
$$




$$
\begin{array}{lr}
r_{t} & =-C^{\prime} H_{t}-1\left(H_{t} b_{t}-h_{t}\right) \\
r_{t}=-C^{-1} H_{t}-1 H_{t} b_{t}+C^{-1} H_{t}-1 h_{t} & \\
r_{t}=-C^{-1}\left(b_{t}-H_{t}-1 h_{t}\right) & \text { ou } \\
H_{t}=P_{t} \text { (vide Fase II) } \\
h_{t}=P_{t} m_{t} \text { (vide Fase IV adiante) } \\
r_{t}=-C^{-1}\left(b_{t}-P_{t}-1 P_{t} m_{t}\right) \\
r_{t}=-C^{-1}\left(b_{t}-m_{t}\right) .
\end{array}
$$

Fase IV: $O$ vetor $h_{t}$ torna-se igual ao produto da matriz de pesos especificados, $P_{t}$, pelo vetor de metas estabelecidas pelos planejadores.

$$
\begin{aligned}
h_{t}= & P_{t} m_{t}-A^{\prime} H_{t+1}\left(b_{t+1}+C r_{t+1}\right)+A^{\prime} h_{t+1} \\
h_{t}= & P_{t} m_{t}-A^{\prime}-H_{t+1}\left\{b_{t+1}+C\left[-C^{-1}\left(b_{t+1}-H_{t+1}^{-1} h_{t+1}\right)\right]\right\}+ \\
& A^{\prime} h_{t+1} \\
h_{t}= & P_{t} m_{t}-A^{\prime} H_{t+1}\left\{b_{t+1}-b_{t+1}+H_{t+1}^{-1} h_{t+1}\right\}+A^{\prime} h_{t+1} \\
h_{t}= & P_{t} m_{t}-A^{\prime} h_{t+1}+A^{\prime} h_{t+1} \\
h_{t}= & P_{t} m_{t}
\end{aligned}
$$

FASE V: Esta fase mostra que, dados os controles "ótimos" $X_{t}$, os valores calculados das variåveis endógenas serão sempre iguais às metas:

$$
\begin{aligned}
& Y_{t}=\left(A+C L_{t}\right) Y_{t-1}+C r_{t+1} b_{t} \\
& Y_{t}=\left[A+C\left(-C^{\prime} A\right)\right] Y_{t-1}+C r_{t}+b_{t}
\end{aligned}
$$




$$
\begin{aligned}
& Y_{t}=[A-A] Y_{t-1}+C r_{t}+b_{t} \\
& Y_{t}=C r_{t}+b_{t}
\end{aligned}
$$

substituindo $r_{t}$, vem:

$$
\begin{aligned}
& Y_{t}=C-C^{-1}\left(b_{t}-H_{t}^{-1} h_{t}\right)+b_{t} \\
& Y_{t}=-C C^{-1} b_{t}+C C^{-1} H_{t}^{-1} h_{t}+b_{t} \\
& Y_{t}=-b_{t}+H_{t}^{-1} h_{t}+b_{t} \\
& Y_{t}=H_{t}^{-1} h_{t}
\end{aligned}
$$

como $H_{t}=P_{t}$ e $h_{t}=P_{t} m_{t}$, tem-se

$$
\begin{aligned}
& Y_{t}=P_{t}^{-1} P_{t} m_{t} \\
& Y_{t}=m_{t} .
\end{aligned}
$$

Esta fase mostra que existe perfeita controlabilidade do sistema; as variáveis endógenas podem ser completamente dirigidas pelas variáveis de controle a atingir quaisquer niveis que se deseje em apenas um perlodo de tempo.

Para melhor elucidar este fato, considere o modelo:

$$
Y_{t}=A Y_{t-1}+C X_{t}+b_{t}
$$

onde é especificado um vetor de metas $m_{t}$ para as variáveis-estado e a matriz $\mathrm{C}$ é não-singular. Então, adotando

$$
\begin{aligned}
& X_{t}=C^{-1}\left(m_{t}-A Y_{t-1}-b_{t}\right), \text { vem: } \\
& Y_{t}=A Y_{t-1}+C\left[C^{-1}\left(m_{t}-A Y_{t-1}-b_{t}\right)\right]+b_{t} \\
& Y_{t}=m_{t} .
\end{aligned}
$$

Como se vê, existe uma solução única para metas e controles. Não hâ, 
neste caso, o que otimizar e a solução do problema pode ser obtida resolvendo-se o sistema (16).

Fase VI: Para qualquer matriz de pesos, $P_{t}$, a trajetória ótima permanece a mesma, pois $L_{t}$ é constante e $r_{t}$ independente dos pesos.

$$
X_{t}=L Y_{t-1}+r_{t}
$$

Qualquer exerclcio de simulação, que tenha por objetivo verificar diferentes niveis de controles para diferentes combinaçōes de pesos atribuildos às metas, será infrutffero. Conforme a Fase $V$, estabelecidas as metas, que as variáveis endógenas deverão assumir, os níveis das variáveis de controles ficam determinados pela solução do sistema (16).

Fase VII: Os multiplicadores de Lagrange serão nulos para todo o perlodo de planejamento.

$$
\begin{aligned}
& \lambda_{t}=H_{t} Y_{t}-h_{t} \quad \text { mas, } \\
& H_{t}=P_{t}\left(2^{a} \text { fase }\right) e h_{t}=P_{t} m_{t} \text { (Fase IV) logo: } \\
& \lambda_{t}=P_{t} Y_{t}-P_{t} m_{t}
\end{aligned}
$$

como $Y_{\mathrm{t}}$ é igual às metas estabelecidas (Fase V), tem-se:

$$
\begin{aligned}
& \lambda_{t}=P_{t} m_{t}-P_{t} m_{t} \\
& \lambda_{t}=0 .
\end{aligned}
$$

Esta fase evidencia o significado dos multiplicadores de Lagrange. Conforme visto, eles representam o custo marginal dos desvios entre os nlveis observados das variáveis-estado e suas metas. Aqui, as variáveis co-estado sāo todas nulas, pois as metas são plenamente atingidas pela solução do sistema (16). 
Deve-se annda avaliar quais os aspectos metodológicos que um MLQ deve obedecer a fim de que uma soluçāo ótima seja obtida por meio das sete fases de cálculos apresentadas.

Como todo o desenvolvimento do algoritmo de soluçāo depende da matriz $\left(\mathrm{C}^{\prime} \mathrm{H}_{4} \mathrm{C}\right)$, é importante que se explore os fatores que influenciam a singularidade ou não-singularidade desta matriz. Para tal exame, dois teoremas são de fundamental importância.

- Teorema I: Seja uma matriz simétrica $H$ de ordem $n \times m$ com posto $r \leq n$. Existe uma matriz $Q$ de ordem $r \times n$, de posto completo $r$, tal que $Q^{\prime} \mathrm{Q}=\mathrm{H}^{3}{ }^{3}$

- Teorema II: O posto de uma matriz qualquer não se altera quando ela for pré ou pós-multiplicada por uma matriz nãosingular. ${ }^{4}$

No MLQ, a matriz H é uma matriz diagonal de ordem $p$, logo é simétrica. Pelo teorema I, existe uma matriz $Q$, de ordem $p$ e posto completo, tal que $Q^{\prime} \mathrm{Q}=\mathrm{H}$. Pode-se, portanto, escrever:

$C^{\prime} H_{t} C=C^{\prime} Q^{\prime} Q C$ onde $Q^{-1}$ existe.

Fazendo $\mathrm{QC}=\mathrm{B}$ tem-se:

$C^{\prime} Q^{\prime} Q C=B^{\prime} B$

Pelo teorema II, $B^{\prime} B$ tem o mesmo posto de $C$, ou seja: $\rho(B)$ $=\rho(C)$. Logo, se o posto de $C$ for completo, então $B^{\prime} B$ será não-singular e, conseqüentemente, $\left(\mathrm{C}^{\prime} \mathrm{H}_{t} \mathrm{C}\right)^{-1}$ existe.

Como se vê, a análise metodológica referente à existência do algoritmo de solução, fundamenta-se no exame do posto da matriz de coeficientes $\mathrm{C}$.

Basicamente, duas são as situaçōes que podem ocorrer na formulação de um MLQ. A primeira trata de um modelo no qual o conjun-

3 THEIL (1971, p. 23)

4 THEIL $(1971, p, 12)$ 
to de restriçōes é especificado com as variáveis-estado em menor número do que as variáveis de controle. Considerando-se $p$ variáveis-estado e $q$ variáveis de controle com $p<q$, tem-se a matriz $C$ de ordem $p \times q$, a matriz $H_{t}$ de ordem $p$ e a matriz $\left(C^{\prime} H_{t} C\right)$ de ordem $q$. Mesmo com as matrizes $C$ e $H_{t}$, apresentando posto completo, $\rho(C)=p$ e $\rho\left(H_{t}\right)=p$, a matriz $\left(C^{\prime} H_{t} C\right)$ terá, no máximo, posto igual a $p$. É uma matriz singular e, portanto, o método não apresenta solução. Nesta situação, pode-se igualar $(q-\rho)$ elementos de $X_{t}$ a quaisquer vatores arbitrários e resolver o modelo para os demais. Com os valores iniciais de $X_{t}$, tem-se uma nova configuração do modelo onde a matriz $\mathrm{C}$ é de ordem $\mathrm{p}$ e $\mathrm{q}$, o número de variáveis-estado é igual ao de variáveis de controle. A matriz $C$ poderá ser singular ou não-singular. Se não-singular, o modelo apresentará soluçāo única, condicionada aos (q- $\rho$ ) valores de $X_{t}$. Caso contrário, se $C$ singular, o método não apresentará solução. 5

$\dot{E}$ importante ressaltar que a singularidade da matriz $C$ impede 0 desenvolvimento da primeira fase de cálculo do algoritmo proposto. Contudo, o modelo apresenta soluçāo e outros métodos podem. ser utilizados para resolvê-lo. É nesta fase do processo que se enfrenta a diflcil tarefa de decidir entre dois caminhos disponiveis: a reformulação do modelo (que pode ser feita com a inclusão de novas variáveis-estado) visando a obter $C$ não-singular, ou solucioná-lo por meio de outro método. Esta última alternativa, além de complexa, não propicia a "regra de controle", que é considerada de grande utilidade na previsão de controles futuros, como também não favorece interpretações econômicas de modo transparente, como o algoritmo de soluçāo apresentado.

A segunda situação, que merece destaque, refere-se a um modelo cujo conjunto de restriçōes é especificado com as variáveis-estado em maior número do que as variáveis de controle, ou seja, $p>q$. Para garantir a existência de $\left(\mathrm{C}^{\prime} \mathrm{H}_{\mathrm{t}} \mathrm{C}\right)$ é necessário que as matrizes $\mathrm{C}$ e $H_{t}$ tenham posto completo, isto é, $\rho(C)=q$ e $\rho\left(H_{t}\right)=p$. Caso a matriz C não apresentar posto completo, mais uma vez não será posșível resolver o MLQ pelo seu algoritmo. Neste caso, através da definição de novos controles, pode-se eliminar informações repetidas, diminuindo a ordem de $C$ até que atinja posto completo. Desse modo, pode-se se chegar ao caso de igualdade entre variáveis de controle e variáveis-

5 Considerando-se as matrizes $\mathrm{C}$ e $\mathrm{H}_{\mathrm{t}}$ de ordem $\mathrm{p}$, entăo a matriz (c ${ }^{\prime} \mathrm{HC}$ ) é também de ordem p. Se $\rho(C)=r<p, C$ será singular $\theta$, pelo teorema I, $B^{\prime} B$ também. Assim (C ' $\left.H C\right)$ não admitúrá inversa. 
estado, cuja solução, como visto anteriormente, é única. Logo, o esforço de eliminar as combinaçóes lineares existentes em $C$ tem por objetivo obter uma nova matriz com posto completo e que não admita inversa. Em outras palavras, a matriz de coeficientes das variáveis de controle pode apresentar combinaçōes lineares desde que, ao eliminá-las, consiga-se manter as variáveis-estado em maior número do que as variáveis de controle. Só assim se conseguirá as soluçōes ótimas esperadas.

Em vista dessas considerações, pode-se concluir que para trabalhar com o algoritmo de solução de um MLQ, é essencial que a matriz $\mathrm{C}$ apresente posto completo e seja singular. Em qualquer outro caso, a solução obtida será única, ou única condicionada a determinados valores iniciais para as variáveis de controle. $E$ isso nada mais é, que um processo de simulação, e não de otimização. Assim, um modelo inserido nesse caso particular de solução escapa do principal fundamento de um MLQ; o de encontrar endogenamente os niveis "otimos" de todas as variåveis de polltica envolvidas no modelo, sem qualquer interferência de valores iniciais atribuldos exogenamente a essas variáveis.

É fundamental, portanto, que a matriz de coeficientes das variáveis de controle satisfaça as condiçōes acima, se um MLQ está para ser utilizado em um caminho diferente de um processo de simulação. Isto é, no sentido de avaliar soluções ótimas, como também analisar o custo de caminhós subótimos em programas de estabilização econômica.

Além dessas consideraçōes, na formulação de um MLQ, a especificação de uma funcional-objetivo quadrática e de um conjunto de restriçōes lineares pode ser considerada como uma limitação ao mes mo. Argumenta-se, entretanto, que o uso de funcionais-quadráticas é familiar em modelos econômicos. Elas são consideradas como um caminho razoável na modelagem de desvios entre valores observados e metas propostas. São restritivas, no entanto, pelo fato de serem simétricas, pois tem-se o mesmo custo tanto para os desvios positivos das variáveis-estado em relação às suas metas, quanto para os desvios negativos.

Talvez, uma das mais importantes limitaçōes de um MLQ resida no emprego de modelos lineares para representar o estado do sistema. Isto porque, muitos dos aspectos interessantes do comportamento das relaçōes econômicas surgem da nảo linearidade das relações funcio- 
nais envolvidas. As ferramentas anallticas utilizadas no tratamento de sistemas dinâmicos nāo lineares são ainda insuficientes no cômputo de trajetớrias ótimas. Assim, semelhantemente à simulação, toma-se necessário analisar os resultados computacionais para se ter uma melhor percepção do quanto se pode confiar em modelos lineares, como meio de se obter políticas econômicas ótimas.

\section{2 - VARIANTES DE MODELOS LINEARES QUADRÁTICOS}

Entre as alterações que os MLQs podem apresentar, merecem destaque os modelos não lineares, os estocásticos e os que envolvem metas para as variáveis de controle.

Quando o sistema em consideração for representado por um modelo nāo-linear, o processo de resolução, anteriormente exposto, nâu sofre modificaçōes. Para que o algoritmo seja utilizado é requerido apenas que o conjunto de restrições receba algum processo de linearização. ${ }^{6}$

Como se sabe, a linearização de modelos cria dificuldades para predições, principalmente para os períodos que não são imediatamente próximos do ponto no qual o modelo foi linearizado.

Como as soluçōes de controle ótimo envolvem perlodos de tempo além do ponto de linearização, os pontos estimados poderão diferir, em muito, dos valores esperados. Nesses casos, é difícil saber como esses desvios se desenvolvem no decorrer do tempo. Sob esse aspecto, as soluções ótimas obtidas com modelos linearizados são inferiores às alcançadas com os não lineares.

Os MLQs estocásticos envolvem elaboraçōes adicionais. Em termos gerais, eles são decompostos em dois problemas distintos. $O$ primeiro deles é um problema de controle cujo conjunto de restrições é um modelo determinístico, isto é:

$$
Y_{t}=A_{t} Y_{t-1}+C X_{t}+b_{t}
$$

segundo problema, no entanto, tem como conjunto de restriçōes um modelo estocástico do tipo:

6 Isso pode ser feilo, por exemplo, atravếs da expansăo de Taylor. 


$$
Y_{t}^{e}=A_{t} Y_{t-1}^{e}+C X_{t}^{e}+\mu_{t}
$$

que representa o desvio entre o vetor aleatório $Y_{t}$ e a trajetória dada pelo modelo determinístico (17). Assim, $Y_{t}^{e}=Y_{t}-\bar{Y}_{t}$ é o vetor das variáveis-estado no tempo $\mathrm{t}$, obtidas com o modelo deterministico.

Significa dizer que existe um desvio entre os valores observados das variáveis-estado $Y_{t}$ e os valores que elas deveriam assumir, quando são aplicados os controles "stimos", $\bar{X}_{t}$, alcançados por meio da solução do modelo determinístico.

O resultado "Śtimo", $\bar{X}_{t}$, é alterado por um vetor estocástico de controles, isto é: $X_{t}^{e}=X_{t}-\bar{X}_{t}$. Isso, com o objetivo de controlar o desvio entre as variáveis-estado, dado por $Y_{t}^{e}=Y_{t}-\bar{Y}_{t}$.

A funcional-objetivo, em modelos desse tipo, representa os valores esperados dos desvios das variáveis-estado. Tem-se então:

$E\left[D_{2}\right]=\frac{1}{2} \sum_{t=t_{0}}^{t_{f}}\left(Y_{t}-m_{t}\right)^{\prime} P_{t}\left(Y_{t}-m_{t}\right)+E\left[\sum_{t=t_{0}}^{t_{f}}\left(Y_{t}^{e}\right)^{\prime} P_{t} Y_{t}^{e_{t}}\right]$

ou mais sinteticamente:

$$
E\left[D_{3}\right]=D_{1}+E\left[D_{2}\right]
$$

onde:

$$
\begin{aligned}
& D_{2}=\sum_{t=t_{0}}^{t}\left(Y_{t}^{e}\right)^{\prime} P_{t}\left(Y_{t}^{e}\right) \\
& D_{1}=\frac{1}{2} \sum_{t=t_{0}}^{t}\left(Y_{t}-m_{t}\right)^{\prime} P_{t}\left(Y_{t}-m_{t}\right) .
\end{aligned}
$$

Nesse tipo de MLQ, as duas etapas de solução consistem em: primeiro, otimizar $\mathrm{D}_{1_{3}}$ como visto anteriormente; segundo, otimizar $E\left[D_{2}\right]$ em relação a $X_{t}^{e}$, dado o conjunto de restriçōes (18). O resultado final será obtido por meio da soma das soluçōes ótimas das variáveis de controle, isto é:

$$
x_{t}=\bar{x}_{t}+x_{t}^{e} \text {. }
$$


O modo de solução de modelos de controle, que incluem um tratamento completo de natureza aleatória do sistema econômico, constitui-se em uma tarefa muito diffcil, se não impossivel. $O$ mais simples tratamento estocástico, por sua vez, permite reduzir esses modelos em deterministicos. A funcional-objetivo, neste caso, é uma função do valor esperado das variáveis-estado; é o denominado problema de "certeza equivalente". Tal problema se resume em uma solução multiperiódica, onde somente os valores para $X_{1}$ são efetivamente aplicados no cálculo dos valores correspondentes aos períodos subseqüentes. Passado o primeiro perlodo dos valores de $x_{2}, x_{3}, \ldots, x_{t f}$ são simultaneamente calculados e $X_{2}$ \&́ aplicado. Esse processo se desenvolve sucessivamente até o último período.

Por último, tem-se os MLQs cuja função objetivo inclui, além da soma dos quadrados dos desvios entre valores observados das variáveis-estado e suas metas, a soma dos quadrados dos desvios entre variáveis de controle e as respectivas metas. Esses modelos são desenvolvidos através do Principio do Máximo de Pontryagin. A função objetivo é dada por.

$D_{4}=\frac{1}{2} \sum_{t=t_{0}}^{t}\left(Y_{t}-m_{t}\right)^{\prime} P_{t}\left(Y_{t}-m_{t}\right)+\left(X_{t}-m_{t}^{c}\right)^{\prime} Q_{t}\left(X_{t}-m_{t}^{c}\right)$

sujeito ao modelo dado por (17).

\section{Onde:}

$m_{t}^{c}=$ vetor $q \times 1$ de metas para as variáveis de controle no perlodo $t$

$Q_{t}=$ matriz diagonal de pesos de ordem q no período $t$.

A matriz $Q_{t}$ é análoga à matriz $P_{t}$. Ela representa o custo de manipulação de determinadas variáveis de controk, em detrimento de outras com o objetivo de levar o sistema ao estado especificado pelo vetor $m_{t}$. As magnitudes comparativas de $P_{t}$ e $Q_{t}$ representam os custos de controlar a economia, relativos aos custos de ter o sistema desviado de sua trajetória "ideal".

7 THEIL (1969, p. 219). 
Nesses modelos, o objetivo é encontrar uma seqüềncia de políticas $X_{t}$, tão próximas a $m_{t}^{c}$ quanto possivel, de modo que aproxime as variáveis-estado, $Y_{t}$, de suas metas pretendidas.

$\dot{E}$ interessante notar que os custos reais dos desvios das variáveis de controle agora estão presentes na função-objetivo. Esse fato impede os planejadores de manipularem as referidas variáveis em qualquer direção, evitando, assimi, o caso particular de solução única. Logo, qualquer análise de viabilidade política e econômica de trajetórias ótimas, obtidas com diferentes matrizes de pesos, será sempre factivel.

Sob o ponto de vista operacional, esses modelos exigem que o usuário tenha grandes habilidades computacionais, já que seu algoritmo de solução é extenso e muito trabalhoso.

PINDYCK (1973) apresenta o desenvolvimento completo de um MLQ com essas características. Ele também especifica e estima um modelo da economia americana que, após simulaçōes e testes, foi utilizado para avaliar os efeitos de diferentes experimentos da política e para demonstrar como um MLQ pode ser empregado em programas de estabilização econômica. $O$ autor salienta ainda, que o cálculo de politicas ótimas, mesmo quando se tem alguma desconfiança sobre 0 modelo utilizado, é vantajoso porque se consegue formar uma idéia da fase adequada de aplicação, tanto de politica fiscal quanto monetária. Como diz Pindyck:

This is probably one of the most important lessons to be learn from our results. (1973:140)

Como se vê, por mais simples que seja um MLQ seus resultados serāo mais precisos do que os obtidos pela simulação: Isso porque a simulaçāo é um processo de tentativa e erro, enquanto que os Modelos de Controle Ótimo identificam o nivel de controle através de soluçōes ótimas. Além disso, esses modelos fornecem relações - regra de controle - entre instrumentos de política e objetivos, bem como interpretações econômicas relevantes.

\section{3 - CONSIDERAÇÕES FINAIS}

Conforme foi visto, todo MLQ apresenta vantagens associadas a dificuldades operacionais. No caso do MLQ não lineares, tem-se a van- 
tagem de se trabalhar normalmente, desde que as restriçọes do modelo sejam linearizadas. Em termos de previsão, contudo, eles apresentam a desvantagem de pontos estimados diferirem de valores esperados, principalmente em pontos que não são imediatamente próximos ao ponto de linearização. A análise dos requisitos metodológicos desse modelo revelou que a existência do algoritmo de solução de pende essencialmente da matriz de coeficientes das variáveis de controle. Isto é, para que um modelo econométrico dinâmico de equações simultâneas represente as restrições de um MLQ, deverá ser especificado de modo que essa matriz tenha posto completo e, por conseguinte, a matriz $\left(\mathrm{C}^{\prime} \mathrm{H}_{\mathrm{t}} \mathrm{C}\right)$ seja não singular. Caso contrário, o algoritmo não apresentará solução. Isso não quer dizer que o modelo não tenha solurção; existem outros métodos para solucioná-lo. Contudo se não for resolvido por seu algoritmo perder-se-á a equação de controle, que constitui-se em uma de suas vantagens.

Tem-se também os MLQs que envolvem restrições às variáveis de controle. Esses modelos requerem do usuário grandes habilidades computacionais, dada a extensão de seu algoritmo de solução. Todavia, com eles, não se chega ao caso de solução única.

Os MLQs estocásticos, por seu turno, possuem soluções complexas, devido às propriedades estocásticas dos parâmetros, e encontram-se em fase de desenvolvimento.

Enquanto as dificuldades operacionais que cercam os. MLQs estocásticos não forem eliminadas, resta a possibilidade de se tratar a incerteza sobre a economia por meio de modelos alternativos em detrimento das propriedades estocásticas dos parâmetros envolvidos.

Nesse contexto, para que a Teoria de Controle Ótimo, assim como a simulação, seja considerada um meio de análise de políticas econômicas alternativas, é imperativo que produza soluçōes computacionais tratáveis. Isso, por enquanto, só é conseguido por meio de modelos determinísticos.

O economista, portanto, deve estar apto para tratar com soluçōes numéricas de modo que possa experimentar os resultados "otimos" com diferentes valores para os parâmetros em sua função-objetivo, ou com diferentes trajetórias temporais para as variáveis exógenas não sujeitas a controle. $E$, como em qualquer outro processo de modelagem, deve enfrentar os resultados apenas como uma aproximação da realidade. 
Finalizando, se obedecidos os requisitos metodológicos, em qualquer tipo de MLQ, os resultados serão ótimos, pelo menos em relação à função-objetivo especificada. É um método que vai além da simulação. Com ele tem-se a certeza de terem sido consideradas todas as combinações possiveis de niveis das variáveis de controle que podem levar o sistema aos objetivos pretendidos, pois a solução é obtida através de um processo de otimizaçāo.

$\mathrm{Na}$ Teoria de Controle Ótimo, a simulação serve como ferramenta adicional. Ela deixa de ser o meio principal de pesquisa de uma triIha de atuação para programas de estabilização econômica. Associada aos MLQs ela serve para analisar diferentes trajetórias "ótimas" e não mais diferentes trajetórias quaisquer. Esse ponto pode ser sintetizado pelas palavras de Pindyck:

Computer simulation is, in fact, often used both to learn more about the dynamic behavior of an econometric model, and to study the effects of different policies. Trial-and-error simulations is, however, an inefficient if not impossible method of amiving at policy plan that is optimal, and a more direct approach is certainly desirable (1973:3).

De qualquer forma, fica evidente a necessidade de se pensar na especificação de modelos econométricos com vistas a sua aplicação na Teoria de Controle Ótimo no que concerne aos requisitos metodológicos impostos pelos MLQs, a fim de se usufruir de seu potencial de análise, dado pela regra de controle e pelos multiplicadores de Lagrange na investigação de caminhos altemativos ótimos e subótimos.

\section{REFERÊNCIAS BIBLIOGRÁFICAS}

ATHANS, N. \& FELB, P. Optimal Control: an Introduction to the Theory and its Aplications. New York, MacGraw-Hill, 1966.

BAUMOL, W.J. Economics Dynamics. New York, MacMillan, 1959. .

BELLMAN, R. Dynamic Programming. New Jersey, Princeton University, 1957.

BRUNNER, K. \& MELTZER, A. Optimal Policies Control Theory and Tecnology Exports. New York, North Holland, 1977. 
CHOW, G.C. Analysis and Control of Dynamic Economic Systems. New York, John Wiley, 1975.

Econometric Analysis by Control Methods. New York, John Wiley, 1981.

. On the Optimal Control of Linear Econometric

Systems With Finite Time Horizont. International Economic

Review, Philadelphia, University of Pennsylvania, 13(1):16-25, Febr, 1972.

CURRIE, D. Macroeconomic Policy Design and Control Theory - a Failed Partnership? Economic Journal Cambridge, England Royal Economic Society. 95 (378):285-306, Jun, 1985.

DALAND, R.T. Estratégia e Estilo de Planejamento Brasileiro.

Rio de Janeiro, Lidador, 1967.

DORFMAN, Robert. An Economic Interpretation of Optimal Control Theory. American Economic Review, Nashville, Tenesee, E.U.A. 59 (5):817-81, Dec, 1969.

FONSECA, M.A.B. \& LOPES, C.M.M. Towards an Employment Policy. Optimurn Control Theory and Input-Output Analysis as Instruments to Planning. Revista de Econometria, UnB, Brasilia, 4 (1):23-40, abr, 1984.

FRIEDMAN, B.M. Methods in Optimization for Economic Stabilization Policy. Amsterdam, North Holland, 1973.

INTRILLIGATOR, M.D. Econometric Models, Techniques and Applications. Amsterdan, North Holland, 1978.

Theory. New Jersey, Prentice-Hill, 1971.

MILLER, R.E. Dynamic Optimization and Economic Applications. New York, MacGraw-Hill, 1979.

MONTEIRO, J.V. Brazilian Economic Policy: an Optimal Control Theory Analisis. Pesquisa e Planejamento Econômico, Rio de Janeiro, IPEA, 25 (2):603-9, dez, 1975.

PINDYCK, R.S. Optimal Planning for Economic Stabilization. Amsterdan, North Holland, 1973.

PINDYCK, \& RUBINFELD, D.L. Econometric Models and Economic Forecasts. Tokyo, MacGraw-Hill, 1976.

SAHOTA, G.S. Brazilian Economic Policy: an Optimal Control Theory Analysis. New York, Praeger Publischers, 1975.

SIMONSEN, M.H. Dinâmica Macroeconômica. São Paulo, MacGraw-Hill; 1983. 
SPULBER, N. \& HOROWITZ, I. Quantitative Economic Policy and Planning: Theory and Models of Economic Control. New York, W.W. Norton, 1976.

THEIL, H. Principles of Econometrics. New York, John Wiley.

THEIL, H. et alii. Investigacion Operativa y Economia Cuantitativa. Barcelona, Gustavo Sili, 1969.

WALSH, GR. Methods Optimization. New York, John Wiley, 1975.

\begin{abstract}
This article aims to verify how the Optimal Control Theory may be introduced in an economic planning process in order to achieve a sequence of "optimum" policies, id est, a vector of policies whose levels will be the result of a process of dynamic optimization. The methodology of a Quadratic Linear Model was analyzed with relevant economic interpretations through "co-estate" variables.

A need of thinking in the specification of econometrics model was made clear aiming at its application in the Optimal Control Theory whit the purpose of using the power of analysis given by the control rule as we ll as by the Lagrange Multupliers, in searching for optimum and sub-optimum wayse.
\end{abstract}

